\title{
Lossless Medical Image Compression by Multilevel Decomposition
}

\author{
K.S. Kang and H.W. Park
}

Lossless image coding is important for medical image compression because any information loss or error caused by the image compression process could affect clinical diagnostic decisions. This paper proposes a lossless compression algorithm for application to medical images that have high spatial correlation. The proposed image compression algorithm uses a multilevel decomposition scheme in conjunction with prediction and classification. In this algorithm, an image is divided into four subimages by subsampling. One subimage is used as a reference to predict the other three subimages. The prediction errors of the three subimages are classified into two or three groups by the characteristics of the reference subimage, and the classified prediction errors are encoded by entropy coding with corresponding code words. These subsampling and classified entropy coding procedures are repeated on the reference subimage in each level, and the reference subimage in the last repetition is encoded by conventional differential pulse code modulation and entropy coding. To verify this proposed algorithm, it was applied to several chest radiographs and computed tomography and magnetic resonance images, and the results were compared with those from well-known lossless compression algorithms. Copyright $\odot 1996$ by W.B. Saunders Company

KEY WORDS: lossless image compression, medical image compression, multilevel decomposition (MLD), prediction coding, classification, picture archiving and communication system (PACS).

$\mathbf{C}$ OMPARED WITH analog radiograph film images, digital images have several advantages, such as flexible image processing, convenient database access, and errorless image replication. However, digital imaging requires a large amount of data. Therefore, various data compression algorithms have been introduced to reduce the amount of data. ${ }^{1}$

A common characteristic of digital images, including medical images, is that neighboring pixels have a high degree of correlation. Most data compression methods aim to reduce spatial redundancy by using this high-correlation property. Data compression methods are usually classified into irreversible (lossy) and reversible (lossless) methods. A lossy scheme can attain very high compression ratio, eg, 10:1 or more, but does not allow exact recovery of the original image. On the other hand, a lossless scheme typically achieves a compression ratio of $2: 1$ to
$4: 1$, depending on the image characteristics and compression algorithms used, but the exact recovery of the original image is guaranteed. ${ }^{2}$ For medical applications, a lossless coding scheme is necessary because any information loss or error during the compression process can affect clinical diagnostic decisions.

In lossless image compression, there are usually two distinct steps, decorrelation and coding. Decorrelation is applied to remove the spatial redundancy from the original image so that the entropy of the decorrelated image can be decreased. The amount of data is not decreased in the decorrelation step. In the coding step, the actual data compression is performed according to the entropy reduction by the decorrelation. A well-known method for lossless coding is variable length coding (VLC), which assigns short code words for highly probable pixel values and longer code words for less probable values. The performance of this coding step is limited by the entropy of the decorrelated image data; this is called Shannon's noiseless coding theorem. ${ }^{3}$

The efficiency of decorrelation is measured in terms of entropy. Let us denote the gray levels of the decorrelated image by $\mathrm{f}_{l}, \mathrm{f}_{2}, \ldots, \mathrm{f}_{k}$, and let the probability of the gray level $\mathrm{f}_{i}$ be given by $P_{i}$. The entropy of the decorrelated image is defined as

$$
H=-\sum_{i=1}^{k} P_{i} \cdot \log _{2} P_{i} .
$$

The entropy $H$ is the lower bound of the average code length obtained by using a VLC such as Huffman coding or Arithmetic coding. ${ }^{3}$

Several decorrelation algorithms for lossless

From the Department of Information and Communication Engineering, Korea Advanced Institute of Science and Technology, Seoul, Korea.

K.S. Kang is now with LG Information \& Communications, Ltd, Korea.

Address repint requests to H.W. Park, PhD, Department of Information and Communication Engineering, Korea Advanced Institute of Science and Technology, 207-43, Cheongryangri, Dongdaemungu, Seoul 130-012, Korea.

Copyright $\odot 1996$ by W.B. Saunders Company

0897-1889/96/0901-0006\$3.00/0 
compression have been introduced, such as differential pulse code modulation (DPCM), 4,5 hierarchical interpolation (HINT) ${ }^{6}$ and prediction/classification. ${ }^{7}$ In the study by Roos et al, ${ }^{8}$ HINT was selected as the best decorrelation method for lossless compression. However, in the paper by Lee et $\mathrm{al}^{7}$ the prediction/ classification method showed the best decorrelation performance, especially for computed tomography (CT) images.

The above three algorithms are commonly based on a predictive decorrelation scheme. DPCM uses the previous pixels or a statistical model of the image to predict current pixels, whereas HINT uses a hierarchical interpolation method to predict pixels. The prediction/ classification method uses an interpolation method for prediction and a classification scheme that divides the prediction error image into two groups according to a classification criterion, eg, absolute slope change. A common idea between the HINT and the prediction/ classification methods is the use of a reference subimage to predict the other subimages. Another similarity can be found in the decomposition of the raw image data. The decomposition in the prediction/classification method is very much similar to that in the three-level HINT method.

This paper proposes a new lossless coding algorithm and compares its performance to those of the above three algorithms. The main structure of the proposed algorithm is based on the prediction/classification algorithm. Therefore, the proposed algorithm is mainly compared with the prediction/classification method. The proposed algorithm can be used for medical imaging systems, eg, a picture archiving and communication system (PACS). ${ }^{9}$

The following sections briefly describe the HINT and prediction/classification algorithms and introduce the proposed algorithm, which is called multilevel decomposition (MLD). See Simulation Results and the comparison study to verify the high performance of the proposed algorithm.

\section{HINT}

HINT is a predictive decorrelation algorithm ${ }^{6}$ in which the encoding and the decoding processes are progressive. In HINT, an image is subsampled at the locations identified by 1 in Fig 1. These pixels are used as reference values to predict other pixels. This subsampled image is coded by DPCM, which is followed by a VLC such as Huffman coding. In the second step, each pixel at location 2 is predicted by a two-dimensional (2D) linear interpolation of the four nearest pixels at location 1 as follows:

$$
\begin{aligned}
P 2(i, j) & =1 / 4[R 1(i, j)+R 1(i, j+1) \\
& +R 1(i+1, j)+R 1(i+1, j+1)],
\end{aligned}
$$

where $P 2(i, j)$ is a predicted value at location 2 , and $R 1(i, j)$ is the real pixel value at location 1 . The difference between the predicted value and the real pixel value is encoded by VLC. Each pixel at location 3 is predicted by a 2-D linear interpolation of the four nearest pixels in the horizontal and the vertical directions, ie, two pixels from location 1 and the other two pixels from location 2. In the case of a five-level HINT as shown in Fig 1A, pixels at locations 4 and 5 are processed by the same procedures as pixels at location 3. The differences between the original and the predicted values in the pixels at locations 3,4 , and 5 are also encoded by VLC.

On the decoder side, the reconstruction process is similar to the encoding process. First, the pixels at location 1 are decoded by VLC, which is followed by DPCM. After the pixels at location 1 are reconstructed, each pixel at location 2 is predicted by a $2 \mathrm{D}$ linear interpolation of the four nearest pixels at location 1 , which were previously reconstructed. To recover the original pixel values at location 2 , these predicted values are added to the corresponding difference values at location 2, which were encoded by VLC in the encoder. This process is repeated
(4)

\begin{tabular}{|l|l|l|l|l|l|l|l|l|l}
\hline 1 & 5 & 3 & 5 & 1 & 5 & 3 & 5 & 1 & \\
\hline 5 & 4 & 5 & 4 & 5 & 4 & 5 & 4 & 5 & \\
\hline 3 & 5 & 2 & 5 & 3 & 5 & 2 & 5 & 3 & \\
\hline 5 & 4 & 5 & 4 & 5 & 4 & 5 & 4 & 5 & \\
\hline 1 & 5 & 3 & 5 & 1 & 5 & 3 & 5 & 1 & \\
\hline 5 & 4 & 5 & 4 & 5 & 4 & 5 & 4 & 5 & \\
\hline 3 & 5 & 2 & 5 & 3 & 5 & 2 & 5 & 3 & \\
\hline 5 & 4 & 5 & 4 & 5 & 4 & 5 & 4 & 5 & \\
\hline 1 & 5 & 3 & 5 & 1 & 5 & 3 & 5 & 1 & \\
\hline & & & & & & & & & \\
\hline
\end{tabular}

B

\begin{tabular}{|l|l|l|l|l|l|l|l|l|l}
\hline 1 & 3 & 1 & 3 & 1 & 3 & 1 & 3 & 1 & \\
\hline 3 & 2 & 3 & 2 & 3 & 2 & 3 & 2 & 3 & \\
\hline 1 & 3 & 1 & 3 & 1 & 3 & 1 & 3 & 1 & \\
\hline 3 & 2 & 3 & 2 & 3 & 2 & 3 & 2 & 3 & \\
\hline 1 & 3 & 1 & 3 & 1 & 3 & 1 & 3 & 1 & \\
\hline 3 & 2 & 3 & 2 & 3 & 2 & 3 & 2 & 3 & \\
\hline 1 & 3 & 1 & 3 & 1 & 3 & 1 & 3 & 1 & \\
\hline 3 & 2 & 3 & 2 & 3 & 2 & 3 & 2 & 3 & \\
\hline 1 & 3 & 1 & 3 & 1 & 3 & 1 & 3 & 1 & \\
\hline & & & & & & & & &
\end{tabular}

Fig 1. Pixel classification for five-level HINT (A) and threelevel HINT (B). Pixels at lower numbers are used to predict pixels at the next higher number. 
for the pixel values at locations 3, 4, and 5, sequentially.

\section{PREDICTION/CLASSIFICATION}

The prediction/classification algorithm introduced by Lee et al, ${ }^{7}$ is based on a predictive decorrelation method used in conjunction with a classification concept. In this algorithm, an image is decomposed into four subimages by subsampling the pixels at even and odd (both row and column) locations as shown in Fig 2, which is similar to three-level HINT. The four decomposed subimages (called reference [R], horizontal [H], vertical [V], and diagonal [D], respectively) are strongly correlated with each other because most images have very high correlation coefficients of around 0.95 between adjacent pixels. ${ }^{1}$

By using this high correlation property, the three subimages $\mathrm{H}, \mathrm{V}$, and $\mathrm{D}$ can be predicted from the reference subimage $\mathrm{R}$ by a onedimensional linear or a one-dimensional thirdorder polynomial interpolation. The prediction error between the predicted subimage and the original subimage is classified into two groups by using the change in the absolute slope of the reference subimage, and the classified errors are encoded by VLC with corresponding optimum code words. The remaining reference subimage is encoded by DPCM followed by VLC.

MLD is also a predictive coding method similar to the HINT and the prediction/ classification algorithms. The basic concept of
MLD is based on the prediction/classification algorithm.

In the MLD algorithm, an image is divided into four subimages, ie, $\mathrm{R}, \mathrm{H}, \mathrm{V}$, and $\mathrm{D}$ subimages, according to their locations as shown in Fig 2, which are the same as in the prediction/ classification method. Because the four decomposed subimages are strongly correlated with each other, the three subimages $\mathrm{H}, \mathrm{V}$, and $\mathrm{D}$ can be predicted from the reference subimage, $\mathrm{R}$, where the predicted subimages are denoted by $H_{p}, V_{p}$, and $D_{p}$, respectively. Two different predictors are used for this prediction, ie, a one-dimensional third-order polynomial interpolator for the horizontal and the vertical predicted subimages, and a 2D linear interpolator for the diagonal predicted subimage as shown in Fig 3, A and B, respectively. The one dimensional third-order polynomial interpolation predictors are

$$
\begin{aligned}
h_{p}(i, j)=\{-r(i, j-1)+ & 9 r(i, j)+9 r(i, j+1) \\
& -r(i, j+2)\} / 16
\end{aligned}
$$

for horizontal predicted subimages and

$$
\begin{aligned}
v_{p}(i, j)=\{-r(i-1, j)+ & 9 r(i, j)+9 r(i+1, j) \\
& -r(i+2, j)\} / 16
\end{aligned}
$$

for vertical predicted subimages. The $2 \mathrm{D}$ linear interpolation predictor is

$$
\begin{aligned}
& d_{p}(i, j)=\{h(i, j)+h(i+1, j)+v(i, j) \\
& +\mathrm{v}(\mathrm{i}, \mathrm{j}+1)\} / 4
\end{aligned}
$$

for diagonal predicted subimages.

\section{Original Image (or reference subimage)}

\begin{tabular}{|l|l|l|l|l|l|}
\hline $\mathrm{r}(1,1)$ & $\mathrm{h}(1,1)$ & $\mathrm{r}(1,2)$ & $\mathrm{h}(1,2)$ & $\mathrm{r}(1,3)$ & $\mathrm{h}(1,3)$ \\
\hline $\mathrm{v}(1,1)$ & $\mathrm{d}(1,1)$ & $\mathrm{v}(1,2)$ & $\mathrm{d}(1,2)$ & $\mathrm{v}(1,3)$ & $\mathrm{d}(1,3)$ \\
\hline $\mathrm{r}(2,1)$ & $\mathrm{h}(2,1)$ & $\mathrm{r}(2,2)$ & $\mathrm{h}(2,2)$ & $\mathrm{r}(2,3)$ & $\mathrm{h}(2,3)$ \\
\hline $\mathrm{v}(2,1)$ & $\mathrm{d}(2,1)$ & $\mathrm{v}(2,2)$ & $\mathrm{d}(2,2)$ & $\mathrm{v}(2,3)$ & $\mathrm{d}(2,3)$ \\
\hline $\mathrm{r}(3,1)$ & $\mathrm{h}(3,1)$ & $\mathrm{r}(3,2)$ & $\mathrm{h}(3,2)$ & $\mathrm{r}(3,3)$ & $\mathrm{h}(3,3)$ \\
\hline $\mathrm{v}(3,1)$ & $\mathrm{d}(3,1)$ & $\mathrm{v}(3,2)$ & $\mathrm{d}(3,2)$ & $\mathrm{v}(3,3)$ & $\mathrm{d}(3,3)$ \\
\hline
\end{tabular}

$\mathrm{R}$ : reference subimage

\begin{tabular}{|l|l|l|}
\hline$r(1,1)$ & $r(1,2)$ & $r(1,3)$ \\
\hline$r(2,1)$ & $r(2,2)$ & $r(2,3)$ \\
\hline$r(3,1)$ & $r(3,2)$ & $r(3,3)$ \\
\hline
\end{tabular}

\begin{tabular}{|l|l|l|}
\hline $\mathrm{h}(1,1)$ & $\mathrm{h}(1,2)$ & $\mathrm{h}(1,3)$ \\
\hline $\mathrm{h}(2,1)$ & $\mathrm{h}(2,2)$ & $\mathrm{h}(2,3)$ \\
\hline $\mathrm{h}(3,1)$ & $\mathrm{h}(3,2)$ & $\mathrm{h}(3,3)$ \\
\hline
\end{tabular}

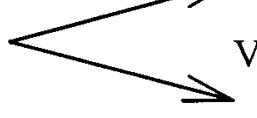

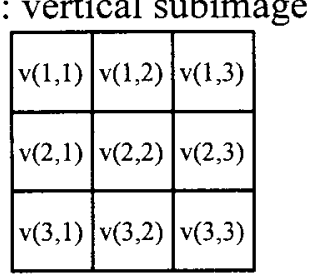

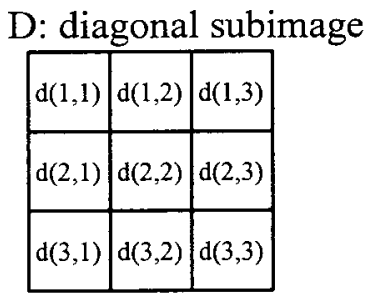

Fig 2. Decomposition by subsampling pixels at even and odd locations. Each subimage has one-half resolution in each direction. 
A

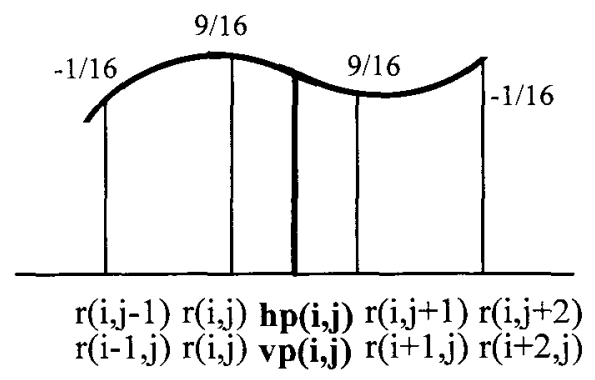

These predictions are not perfect, even though the image is highly correlated and the prediction is very intelligent. Therefore, the prediction error, which is the difference between the original subimage and the predicted subimage should be encoded for lossless compression. The prediction errors for the subimages $H_{d}, V_{d}$, and $D_{d}$ are defined as follows:

$$
\begin{aligned}
& h_{d}(i, j)=h(i, j)-h_{p}(i, j), \\
& v_{d}(i, j)=v(i, j)-v_{p}(i, j),
\end{aligned}
$$

and

$$
d_{d}(i, j)=d(i, j)-d_{p}(i, j) .
$$

In most images including medical images, these prediction errors will be very small, because the four subimages are strongly correlated. These small errors can make the entropy of the prediction error subimages small so that compression performance can be improved. Although this prediction error is small value and less correlated, there is room to reduce statistical redundancy. The prediction error is expected to be small when the corresponding pixel values of the reference subimage, $\mathrm{R}$, change smoothly, whereas the prediction error may be relatively large when the pixel values of $\mathrm{R}$ change rapidly.

$$
{ }_{1 / 4} \mathrm{O}_{\mathrm{h}(\mathrm{i}, \mathrm{j})}
$$

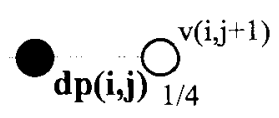

$$
{ }_{1 / 4} \bigcirc_{h(i+1, j)}
$$

Fig 3. Graphical descriptions of two interpolation methods for prediction. (A) One-dimensional third-order polynomial interpolation for $H_{p}$ and $V_{p} ;(B) 2 D$ linear interpolation for $D_{p}$.

By using the above property, each pixel in the prediction error subimages is classified into two or three groups to get the minimum entropy. Two kinds of classification criteria are used. For the horizontal and the vertical prediction error subimages, the absolute slope change of the reference subimage is used. This criterion is the same as that of the prediction/classification algorithm. The absolute slope change are defined as

$$
S_{h}(i, j)=\left|s_{j}-s_{j-1}\right|+\left|s_{j+1}-s_{j}\right|,
$$

where $s_{j}=r(i, j+1)-r(i, j)$ for horizontal subimages and

$$
S_{v}(i, j)=\left|s_{i}-s_{i-1}\right|+\left|s_{i+1}-s_{i}\right|,
$$

where $s_{i}=r(i+1, j)-r(i, j)$ for vertical subimages as shown in Fig 4A. For diagonal subimages, the maximum difference is used as the classification criterion, ie,

$$
S_{d}(i, j)=|\max (i, j)-\min (i, j)|,
$$

where $\max (i, j)=\operatorname{Max}\{h(i, j), h(i+1, j)$, $v(i, j), v(i, j+1)\}$ and $\min (i, j)=\operatorname{Min}\{h(i, j)$, $h(i+1, j), v(i, j), v(i, j+l)\}$ as shown in Fig 4B.

The threshold values of these classification criteria $\left(S_{h}, S_{v}\right.$, and $\left.S_{d}\right)$ are defined experimentally according to the characteristics of the
Fig 4. Classification criteria. (A) Definition of the slope parameter $s_{i}$ for the absolute slope change of $S_{v}$. (B) Classification criterion, $S_{d}$, for the diagonal predicton error.
A

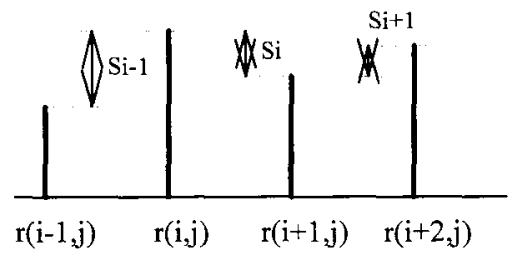

B

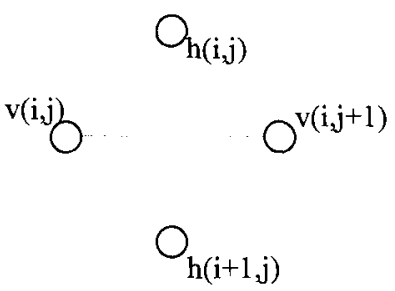

$\max (i, j)=\operatorname{Max}\{h(i, j), h(i+1, j), v(i, j), v(i, j+1)\}$ $\min (i, j)=\operatorname{Min}\{h(i, j), h(j+1, j), v(i, j), v(i, j+1)\}$ 
(4)

\begin{tabular}{|l|l|}
\hline R1 & H1 \\
\hline V1 & D1 \\
\hline
\end{tabular}

B

\begin{tabular}{|c|c|c|}
\hline $\mathbf{R} 2$ & $\mathbf{H} 2$ & $\mathrm{H} 1$ \\
\hline $\mathbf{V} 2$ & $\mathbf{D} 2$ & \\
\hline $\mathrm{V} 1$ & $\mathrm{D} 1$ \\
\hline
\end{tabular}

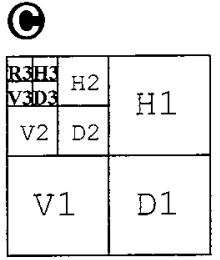

Fig 5. Binary tree-structured multilevel decomposition process. (A) First-level decomposition; (B) second-level decomposition; and (C) third-level decomposition.

reference subimage or the horizontal and the vertical subimages, which will be discussed in the following section. Finally, the classified prediction error subimages are encoded by using VLC, eg, a Huffman ${ }^{10}$ or an arithmetic coding. ${ }^{11.12}$

The above procedures are for a first-level MLD process. To get a second-level MLD process, the reference subimage, $R$, is decomposed into four subimages again, and the above prediction and classification procedures are repeated for the reference subimage as shown in
Fig 5. A higher-level MLD process can be achieved by the same procedures, but the entropy reduction performance gets smaller. Hence, we only tried up to a three-level MLD in the simulation. All the prediction error subimages can be directly encoded by VLC, whereas the final resulting reference subimage, called R3 in a three-level MLD, is decorrelated by DPCM and encoded by VLC.

There are three major differences between the MLD and the prediction/classification algorithms. First, as the name of algorithm indicates, the prediction and classification processes are iterated to the reference subimage in the MLD. Second, for prediction of the pixel values in the diagonal subimage, the MLD algorithm uses a different predictor from that used in the prediction/classification algorithm. In prediction/classification, two (when using the linear interpolator) or four (when using the third-order polynomial interpolator) pixels, which lie on the same diagonal axis in the reference subimages, are used for the one-
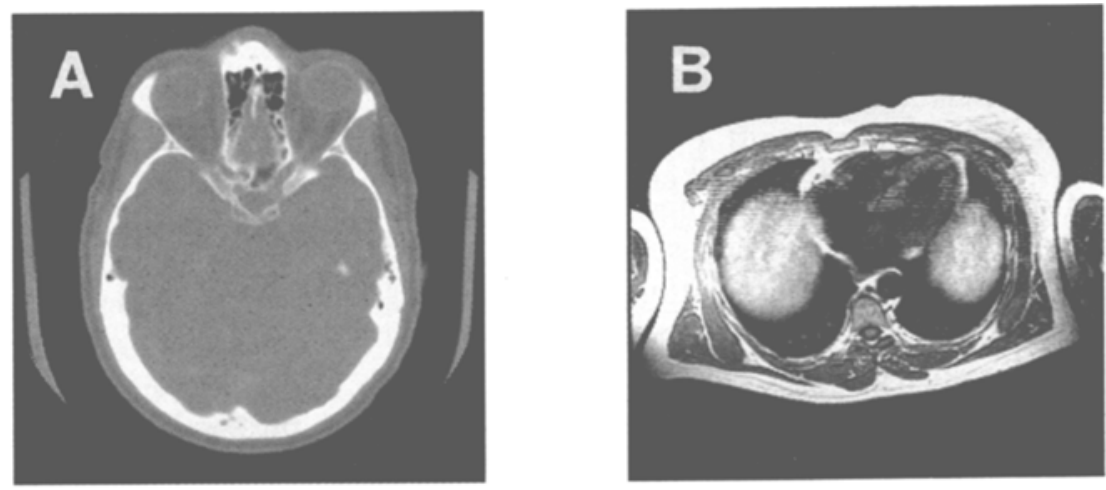

Fig 6. Sample images used in the simulation. (A) One radiograph CT head image, CT 1; (B) MR abdomen image; $(C)$ radiograph chest image; and (D) Lena image.
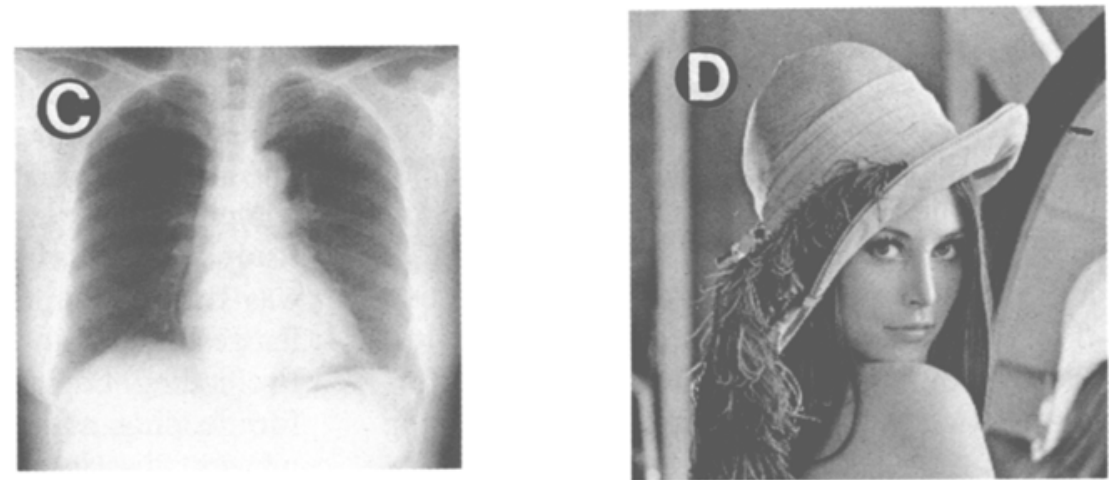

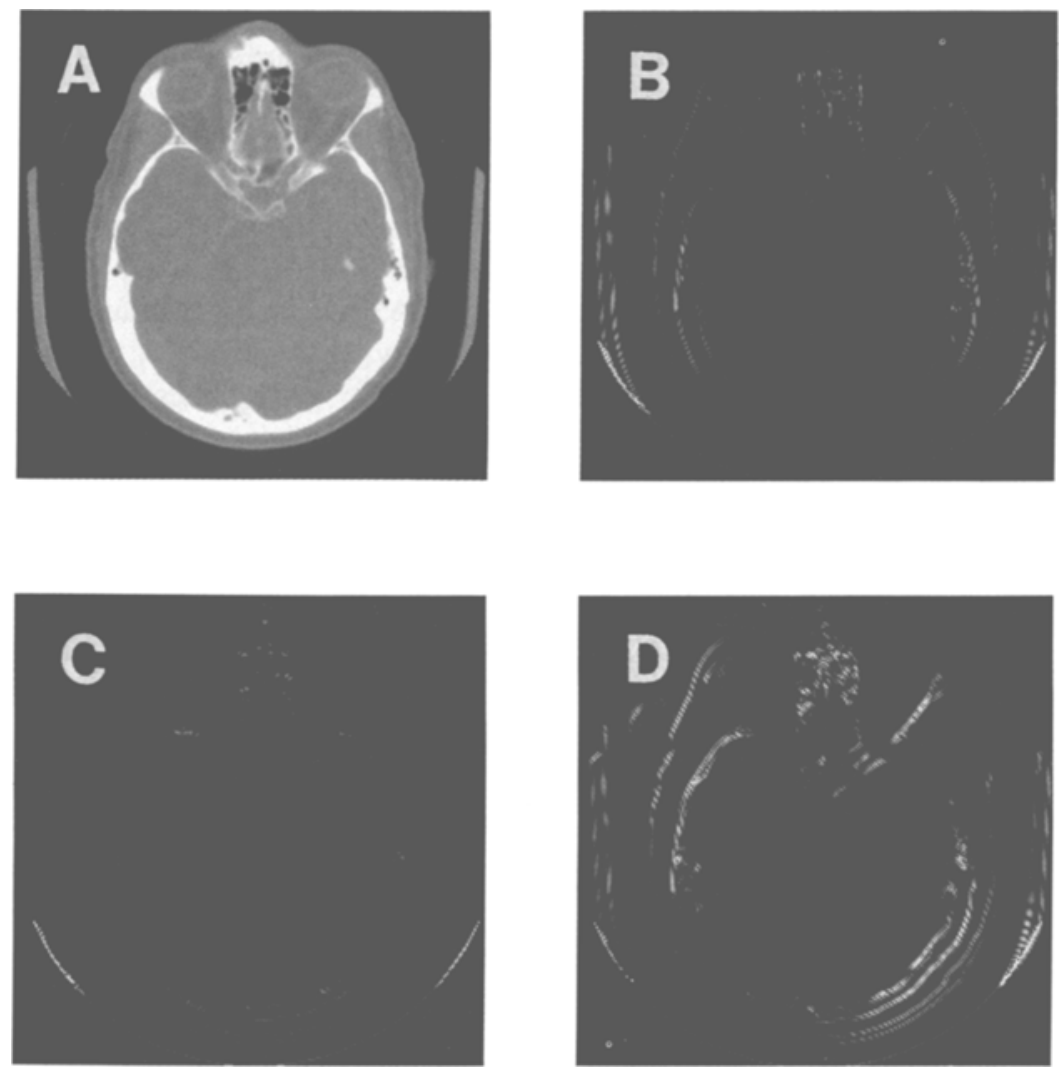

Fig 7. Reference and prediction error subimages of a CT image (CT 1). The prediction error subimages are amplified by a factor of 16. (A) Reference subimage, $R_{\text {; }}(B)$ horizontal prediction error subimage, $H_{d}$ : $(C)$ vertical predicton error subimage, $V_{d}$; and (D) diagonal prediction error subimage, $D_{u}$.

dimensional interpolation. However, in the MLD algorithm, four neighboring pixels in the horizontal and the vertical subimages are used for the 2D linear interpolation as shown in Fig 3B. The prediction procedure for the horizontal and the vertical subimages must be done before prediction of the diagonal subimage because the prediction results for the horizontal and the vertical subimages are used for those of the diagonal subimage during the decoding process. Third, when the MLD algorithm is applied to radiograph CT images, two threshold values are used for classification instead of one. In the radiograph CT images, there is a large background area whose pixel values are zero. Hence, it is reasonable to add one more threshold whose value is zero. Thereafter, the prediction error subimages of the radiograph CT images are classified into three groups by these two threshold values. These three differences allow the improvement in the compression performance, which is described in the following section.

\section{SIMULATION RESULTS}

Computer simulation has been performed to verify the proposed compression algorithm mainly for medical imaging applications. Sample images for simulation include five radiograph CT head images with 512- $\times$ 512-pixel, 12 bit/pixel resolution; one magnetic resonance (MR) abdomen image with $256-\times 256$-pixel, 12 bit/pixel resolution; one. radiograph chest image with 1,024- $\times$ 1,024-pixel, $12 \mathrm{bit} /$ pixel resolution, and the well-known Lena image with 512- $\times$ 512-pixel, 8 bit/pixel resolution, as shown in Fig 6. The radiograph CT images are transverse slices at the forehead level, and the MR abdomen image is also a transverse slice.

A three-level MLD algorithm was used to compress these images in this simulation. In a study by Roos et al, ${ }^{8}$ they showed that HINT was the most appropriate lossless intraframe image compression method from among predictive coding, Laplacian pyramid coding, ${ }^{13}$ transform coding, and HINT. However, for medical images, the prediction/classification method 
Table 1. Total Entropy of Original Sample Images and Their Compression Results

\begin{tabular}{lcccc}
\hline $\begin{array}{c}\text { Image } \\
\text { Name }\end{array}$ & $\begin{array}{c}\text { Original } \\
\text { lmage }\end{array}$ & $\begin{array}{c}\text { HINT } \\
\text { (three-level) }\end{array}$ & $\begin{array}{c}\text { Prediction/ } \\
\text { Classification }\end{array}$ & $\begin{array}{c}\text { MLD } \\
\text { (three-level) }\end{array}$ \\
\hline CT 1 & 8.008407 & 4.457491 & 3.972192 & 3.541023 \\
CT 2 & 7.564907 & 4.280935 & 3.820697 & 3.373437 \\
CT 3 & 7.238710 & 4.147773 & 3.715939 & 3.265292 \\
CT 4 & 7.253867 & 4.139299 & 3.688210 & 3.240012 \\
CT 5 & 7.266698 & 4.035304 & 3.549953 & 3.106719 \\
MR 1 & 8.189445 & 6.558503 & 6.413804 & 6.205520 \\
Radiograph 1 & 10.008707 & 5.211357 & 5.167589 & 4.886617 \\
Lena & 7.445071 & 4.473294 & 4.349309 & 4.258264 \\
\hline
\end{tabular}

showed better performance than HINT in the study by Lee et al. ${ }^{7}$ Therefore, the three-level MLD algorithm was compared with the HINT and the prediction/classification algorithms.

The DPCM algorithm, which was recommended in the Joint Photographic Experts Group (JPEG) ${ }^{14}$ was used as the decorrelation method for the reference subimage in HINT and Prediction/Classification and also for the final reference subimage in MLD. Three-level HINT was adopted for this comparison because the decorrelation process of three-level HINT is similar to that of prediction/classification. In Lee's prediction/classification algorithm, there is just one threshold value for classification of all images, including CT images. However, in our MLD algorithm, there are two threshold values for CT images and one threshold value for other images because CT images have a large background with zero value, which is a characteristic of backprojected images.

Figure 7 shows the reference subimage and the prediction error subimages $H_{d}, V_{d}$, and $D_{d}$ given by equations $4 a, 4 b$, and $4 c$ for first-level MLD. The reference subimage of Fig 7A is decomposed into four subimages again for the next level MLD. These subimages are predicted and classified by the same process as that used for first-level MLD.

Table 1 and Fig 8 show the overall entropy of the sample images using different lossless compression algorithms. The second column of Table 1 lists the original entropy of each test image and the third column lists the entropy of the image decorrelated by three-level HINT. The fourth column shows the entropy of the image decorrelated by a prediction/classification algorithm using a one-dimensional thirdorder polynomial interpolator for prediction. In

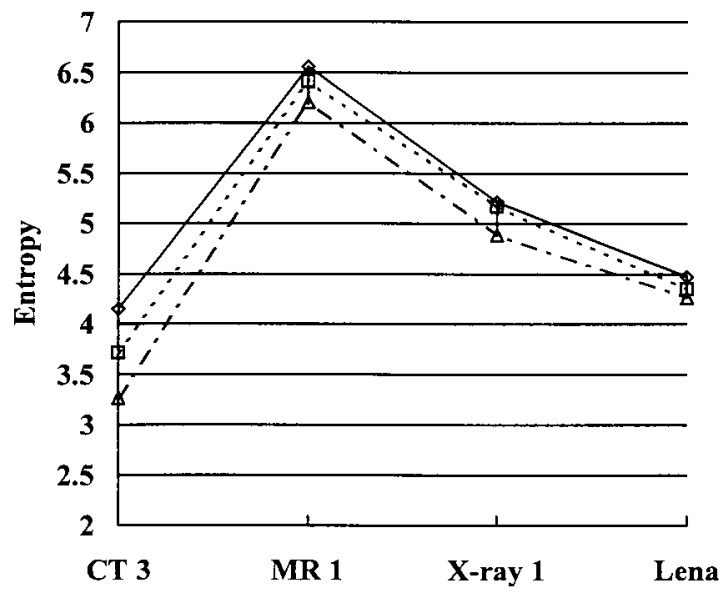

Fig 8. Entropy comparison of the lossless compression algorithms for the sample images. ( $)$, HINT; $(\square)$, P\&C; $(\triangle)$, three-level MLD.

the prediction/classification algorithm, the result using the one-dimensional linear interpolator was poor in comparison with that obtained using the one-dimensional third-order polynomial interpolator. Consequently, the result using the one-dimensional third-order polynomial interpolator is presented here. The last column lists the entropy by three-level MLD. All the entropies in this paper were calculated as in equation 1 . In Table 1, the entropy reduction is small for the Lena image, but large for the medical images, especially the CT images. Figure 8 shows that the entropy of the images decorrelated by three-level MLD is lowest.

In CT images, the entropy differences between the results of three-level MLD and those of prediction/classification range from 0.431 to 0.451 as shown in Table 2. These entropy differences result from the three major improvements that were made by MLD in comparison with prediction/classification and described in the section entitled "MLD." The first improvement is caused by the iteration process of MLD.

Table 2. Entropy Difference Between Prediction / Classification and Three-Level MLD for CT Images

\begin{tabular}{lcccc}
\hline $\begin{array}{c}\text { Image } \\
\text { Name }\end{array}$ & $\begin{array}{c}\text { Prediction/ } \\
\text { Classification }\end{array}$ & $\begin{array}{c}\text { Three-leve } \\
\text { MLD (two } \\
\text { thresholds })\end{array}$ & $\begin{array}{c}\text { Entropy } \\
\text { Difference }\end{array}$ & $\begin{array}{c}\text { Improvement } \\
(\%)\end{array}$ \\
\hline CT 1 & 3.972192 & 3.541023 & 0.431169 & 10.86 \\
CT 2 & 3.820697 & 3.373437 & 0.447260 & 11.71 \\
CT 3 & 3.715939 & 3.265292 & 0.450647 & 12.13 \\
CT 4 & 3.688210 & 3.240012 & 0.448198 & 12.15 \\
CT 5 & 3.549953 & 3.106719 & 0.443234 & 12.49 \\
\hline
\end{tabular}


Table 3. Entropy Decrease for Iterated MLD

\begin{tabular}{|c|c|c|c|c|c|}
\hline $\begin{array}{l}\text { Image } \\
\text { Name }\end{array}$ & $\begin{array}{l}\text { Original } \\
\text { Entropy }\end{array}$ & $\begin{array}{l}\text { One-Level } \\
\text { MLD Entropy }\end{array}$ & $\begin{array}{l}\text { Two-Level } \\
\text { MLD Entropy }\end{array}$ & $\begin{array}{l}\text { Three-Level } \\
\text { MLD Entropy }\end{array}$ & $\begin{array}{c}\text { Three-Level MLD } \\
\text { Compression } \\
\text { Ratio }\end{array}$ \\
\hline CT 1 & 8.008407 & 3.756706 & 3.589275 & 3.541023 & 3.388851 \\
\hline CT 2 & 7.564907 & 3.592826 & 3.423678 & 3.373437 & 3.557203 \\
\hline CT 3 & 7.238710 & 3.484069 & 3.316349 & 3.265292 & 3.675016 \\
\hline CT 4 & 7.253867 & 3.463784 & 3.292239 & 3.240012 & 3.703690 \\
\hline MR 1 & 8.189445 & 6.399594 & 6.261566 & 6.205520 & 1.933762 \\
\hline Radiograph 1 & 10.008707 & 5.003214 & 4.910637 & 4.886617 & 2.455687 \\
\hline Lena & 7.445071 & 4.340445 & 4.274824 & 4.258264 & 1.878700 \\
\hline
\end{tabular}

The CT images have two threshold values.

Table 3 shows the trend in entropy decrease caused by the iteration process. The final column in Table 3 shows the compression ratios, ie, the ratios of the original bits to the entropies from three-level MLD.

The second improvement is caused by the predictor for the diagonal subimages. MLD uses the 2D linear interpolation predictor of equation $3 \mathrm{c}$, whereas prediction/classification uses a one-dimensional third-order polynomial interpolation predictor. Table 4 shows the effect of different predictors for the diagonal subimages. Here, the first-level MLD method using one threshold value is the same as prediction/ classification method except for the predictor for the diagonal subimages. As shown in Table 4 , the 2D linear interpolator is slightly better than the one-dimensional third-order polynomial interpolator for prediction of diagonal subimages, with entropy improvements in the range of 0.019 to 0.03 . The final improvement comes from using two threshold values instead of one when the classification error subimages of the CT image are classified. The third column of Table 3 and the second column of Table 4 show the entropies for MLD using two thresh-

Table 4. Comparison of the Entropy for Different Predictors for Diagonal Subimages

\begin{tabular}{cccc}
\hline $\begin{array}{l}\text { Image } \\
\text { Name }\end{array}$ & $\begin{array}{c}\text { One-Level } \\
\text { MLDWith } \\
\text { One Threshold }\end{array}$ & $\begin{array}{c}\text { Prediction/ } \\
\text { Classification }\end{array}$ & $\begin{array}{c}\text { Entropy } \\
\text { Difference }\end{array}$ \\
\hline CT 1 & 3.953440 & 3.972192 & 0.018752 \\
CT 2 & 3.794386 & 3.820697 & 0.026311 \\
CT 3 & 3.685160 & 3.715939 & 0.030779 \\
CT 4 & 3.658418 & 3.688210 & 0.029792 \\
CT 5 & 3.519634 & 3.549953 & 0.030319 \\
\hline
\end{tabular}

One-level MLD uses a 2D linear interpolation predictor, whereas prediction/classification uses a third-order polynomial interpolation predictor. old values and MLD using one threshold value, respectively, for CT images. The entropy improvement obtained by using two threshold values ranges from 0.180 to 0.202 .

In the third CT image (CT 3), eg, the threelevel iteration MLD procedure results in an entropy reduction of 0.219 , and use of the 2D linear interpolation predictor for the diagonal subimages results in an entropy reduction of 0.031 . Finally, the use of two threshold values results in an entropy reduction of 0.201 . Therefore, the total entropy reduction from the prediction/classification algorithm is 0.451 , which corresponds to the value in Tabel 2 .

When the prediction errors of an image are classified into two or three groups, the decision about threshold values is important. In CT images, one threshold value is always zero because the large background area has a zero value. To find the other threshold value, trend of the entropy as a function of the threshold value is analyzed as shown in Figs 9, 10, 11, and 12. This analysis shows that the threshold value

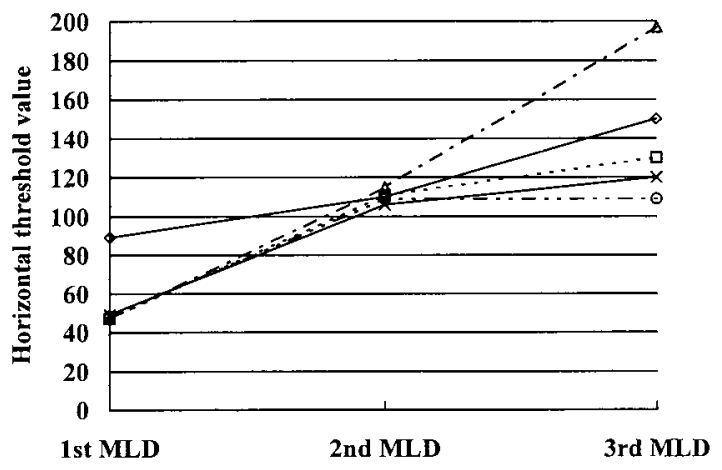

Fig 9. Optimum threshold values for each level to classify horizontal subimages of CT images. $(\diamond), \mathrm{CT} 1 \mathrm{H}$; $(\square)$, CT2 H; $(\triangle)$, СT3 H; $(\times)$, CT4 H; (O), СT5 H. 


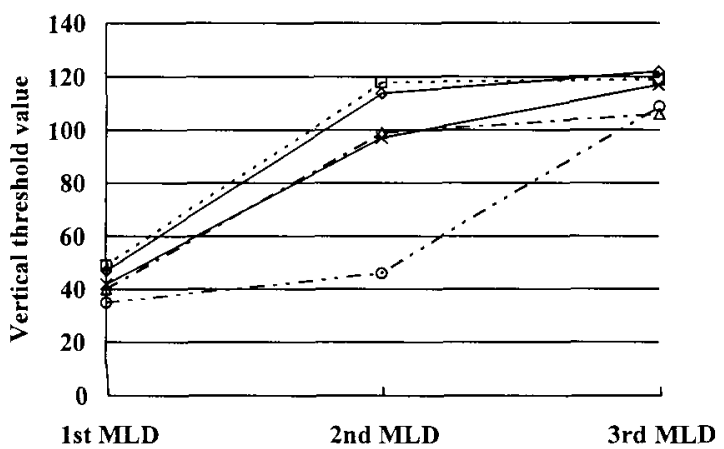

Fig 10. Optimum threshold values for each level to classify vertical subimages of $\mathrm{CT}$ images. $(\diamond), \mathrm{CT} 1 \mathrm{~V} ;(\square), \mathrm{CT} 2 \mathrm{~V} ;(\triangle)$, CT3 V: $(\times)$, CT4 V; (O), CT5V.

increases with the level of MLD because the correlation of the reference subimage gets smaller for higher-level MLD. In Figs 9, 10, and 11 , the threshold values of first-level MLD are obtained from the reference subimage R1 in Fig 5, whereas those of second- and third-level MLD are obtained from R2 and R3 in Fig 5, respectively.

Figure 12 shows the relation between the entropy and the threshold values for a horizontal subimage of first-level MLD, which corresponds to the left part of Fig 9. In Fig 12, the entropy graphs are nicely parabolic near the minimum and, because the parabolas are not sharp, an optimum threshold corresponding to a minimum entropy can be approximately in the range of 40 to 100 without severe entropy loss in this simulation. For example, in Fig 9, the optimum horizontal threshold value for CT 1 by first-level MLD is 90. However, even if we change this threshold value to 50 , the resulting

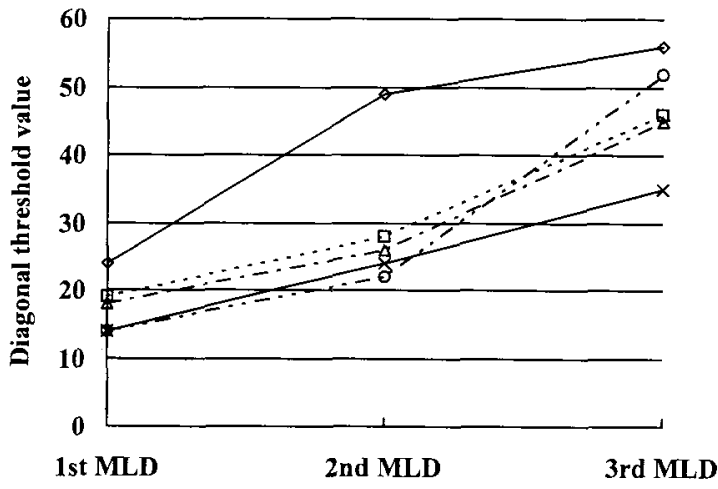

Fig 11. Optimum threshold values for each level to classify diagonal subimages of CT images. $(\backslash), C T 1 D_{;}(\square), C T 2 D ;(\triangle)$, CT3 D; $(\times)$, CT4 D; (O), CT5 D

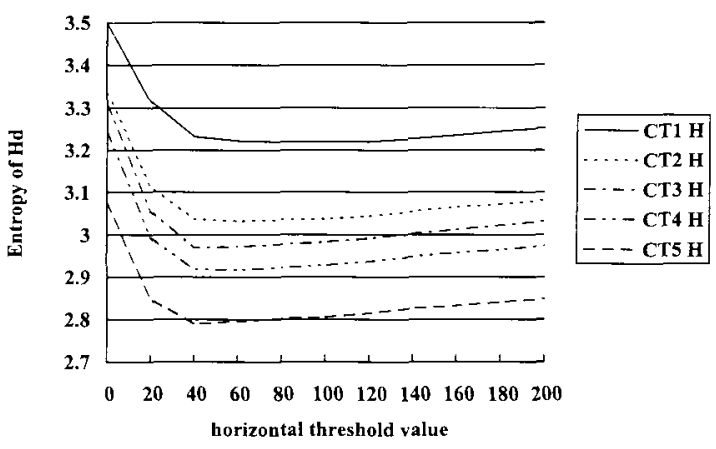

Fig 12. Relation between the entropy of $H_{d}$ and the horizontal threshold values for first-level MLD of CT images.

entropy difference will be only about 0.01 . All the other CT images have the same parabolic entropy distribution. In Figs 9, 10, and 11, another trend of the entropy as a function of the threshold values can be found; the threshold values are clustered around some value for each level and each direction. Using these properties, threshold values for each level and each subimage can be predetermined according to each image type; eg, we can simulate a number of radiograph CT images of transverse slices at the forehead level and predetermine the threshold values for each level and each subimage. In this way, we can save the processing time necessary to determine the threshold values with only a small decrease in performance.

\section{CONCLUSION}

In this paper, a new lossless coding algorithm was proposed for medical image compression, in particular for CT images. The idea of the new algorithm is based on the predicton/classification algorithm, ${ }^{7}$ which has shown the best entropy reduction performance for medical images. Three major improvements are added to the prediction/classification algorithm to achieve high compression performance. The improvements are the iterated process, a 2D linear interpolation predictor for diagonal subimages, and the use of two threshold values to classify the prediction error subimages of CT images.

The proposed algorithm showed an entropy reduction of about 0.45 compared with the prediction/classification algorithm. This corresponds to $10.86 \%$ to $12.46 \%$ more entropy reduction. This algorithm shows good perfor- 
mance for an image with a noticeable edge because the effect of classification will be maximized for that kind of image. Also, it shows good performance for highly correlated images because the effect of prediction depends on the correlation property. A disadvantage of the proposed algorithm is the weakness in the commu- nication channel noise because of the iterated process and the prediction for the horizontal, the vertical, and the diagonal subimages; ie, if some data are lost or changed to different values during transmission, this error will be propagated to the next subimages to be predicted or to the next interation process.

\section{REFERENCES}

1. Jain AK: Image data compression: A review. Proc IEEE 69:349-389, 1981

2. Das $\mathbf{M}$, Burgett $\mathrm{S}$ : Lossless compression of medical images using two-dimensional multiplicative autoregressive models. IEEE Trans Med Imaging MI-12:721-726, 1993

3. Gallager RG: Information Theory and Reliable Communication. New York, NY, Wiley, 1968

4. Zschunke W: DPCM picture coding with adaptive prediction. IEEE Trans Commun COM-25:1295-1302, 1977

5. O'Neil JB Jr: Differential pulse-code modulation (DPCM) with entropy coding. IEEE Trans Informat Theory IT-21:167-174, 1976

6. Endoh T, Yamazaki Y: Progressive coding scheme for multilevel images. Proc Pic Cod Symp 1986, 21-22

7. Lee HS, Kim Y, Oh S: Lossless compression of medical images by prediction and classification. Opt Eng 33:160-166, 1994

8. Roos P, Viergrever MA, Van Dijke MC, et al: Revers- ible intraframe compression of medical images. IEEE Trans Med Imaging MI-7:328-336, 1988

9. Haynor DR, Smith DV, Park HW, et al: Hardware and software requirements for a picture archiving and communication system's diagnostic workstation. J Digit Imaging 5:107-117, 1992

10. Hankamer M: A modified Huffman procedure with reduced memory requirement. IEEE Trans Commun COM27:930-932, 1979

11. Rissanen J, Langdon GG Jr: Arithmetic coding. IBM J Res Dev 23:149-162, 1979

12. Langdon GG Jr, Rissanen $\mathbf{J}$ : Compression of blackwhite images with arithmetic coding: IEEE Trans Commun COM-29:858-867, 1981

13. Burt PJ, Adelson EH: The Laplacian pyramid as a compact image code. IEEE Trans Commun COM-31:532540,1983

14. Pennebaker WB, Mitchell JL: JPEG Still Image Data Compression Standard. New York, NY, Van Nostrand Reinhold, 1993 